\title{
Análise da importância dos marcos anatômicos para a colocação de mini-implantes ortodônticos
}

Analysis of the importance of anatomical landmarks for the placement of orthodontic miniimplants

Análisis de la importancia de los hitos anatómicos para la colocación de miniimplantes de ortodoncia

Maria Renata Alves de Araújo ORCID: https://orcid.org/0000-0001-8845-0767 State University of Paraíba, Brazil E-mail: renataalves914@gmail.com

Andreza Mirelly de Queiroz ORCID: https://orcid.org/0000-0002-3876-2399 State University of Paraíba, Brazil E-mail: amqueiroz07@gmail.com

Fernanda Kelly Costa Tito

ORCID: https://orcid.org/0000-0001-7481-2587 State University of Paraíba, Brazil

E-mail: ffernandacosttaa@gmail.com

Matheus Harllen Gonçalves Veríssimo ORCID: https://orcid.org/0000-0003-2845-4832 State University of Paraíba, Brazil E-mail: matheusharllen@gmail.com

Suzie Clara da Silva Marques ORCID: https://orcid.org/0000-0002-7598-6260 State University of Paraíba, Brazil E-mail: suzieclara08@gmail.com

Tallys da Rocha Borges Leal ORCID: https://orcid.org/0000-0002-6718-759X State University of Paraíba, Brazil E-mail: leal.odontotallys@gmail.com

Tauany Maria da Rocha Borges Leal ORCID: https://orcid.org/0000-0003-4521-4144 State University of Paraíba, Brazil E-mail: tauanyleal17@gmail.com

Thaís Santos de Souza

ORCID: https://orcid.org/0000-0003-4301-9978 State University of Paraíba, Brazil E-mail: thaissouza5086@gmail.com

\section{Resumo}

Introdução: Os mini-implantes representam um grande avanço na Odontologia para auxiliar no tratamento ortodôntico, também denominados de micro implantes e micro parafusos são conhecidos como dispositivos de ancoragem temporária. Objetivos: Realizar uma revisão integrativa da literatura, com base na análise dos marcos anatômicos enfatizando a importância do estudo da anatomia na colocação dos mini-implantes. Metodologia: Trata-se de uma revisão integrativa da literatura de natureza qualitativa realizada a partir da pergunta norteadora: Quais alterações morfofisiológicas interferem no sucesso de mini-implantes em tratamentos ortodônticos? A base de dados foram: $U$. $S$. National Library of Medicine (NLM - PubMed), utilizando os seguintes descritores (MeSHterms): Anatomy; Miniimplants. Os critérios de inclusão foram artigos publicados em inglês, português e espanhol; publicações entre janeiro de 2000 e janeiro de 2021 e que abordavam a temática de maneira satisfatória. Foram excluídos artigos que não atendiam aos critérios de inclusão. Foram encontrados ao todo 198 trabalhos na íntegra; destes, 3 artigos encontravam-se duplicados nas estratégias de busca, totalizando, assim, 8 selecionados. Resultados e Discussão: Constatou-se que é necessária a análise das alterações morfofisiológicas que podem ser encontradas com a utilização de mini-implantes. Também foi observado que o tamanho dos mini-implantes influencia no aumento da ancoragem mecânica, assim como na incidência dos seios paranasais, concluindo que os principais fatores determinantes na ancoragem óssea são a densidade óssea e profundidade de colocação. A utilização de mini-implantes no palato não apresenta interferência nos movimentos dentários, além de ter osso suficiente, possibilitando a inserção do mini-implante. Os autores ainda relatam 
que existem múltiplas razões para a falha ou a perda completa de um mini-implante, como a periodontite e periimplantite, causando infecção de tecidos moles e duros, principalmente quando a higiene oral inadequada ou controle pós-cirúrgico deficiente persiste. Considerações finais: O conhecimento e domínio das estruturas anatômicas de cabeça e pescoço bem como as alterações morfofisiológicas podem influenciar na implantação dos mini-implantes ortodônticos, contribui para o alcance do sucesso clínico, diminuindo efeitos colaterais e o tempo de tratamento é reduzido.

Palavras-chave: Anatomia; Mini-implantes; Ortodontia.

\begin{abstract}
Introduction: Mini-implants represent a great advance in Dentistry to assist in orthodontic treatment, also called micro implants and micro screws are known as temporary anchorage devices. Objectives: To carry out an integrative literature review, based on the analysis of anatomical landmarks, emphasizing the importance of studying anatomy when placing mini-implants. Methodology: This is an integrative literature review of a qualitative nature based on the guiding question: What morphophysiological changes interfere with the success of mini-implants in orthodontic treatments? The database was: National Library of Medicine of the USA (NLM - PubMed), using the following descriptors (MeSHterms): Anatomy; Mini-implants. Inclusion criteria were published in English, Portuguese and Spanish; publications between January 2000 and January 2021 and that addressed the subject in a satisfactory manner. Articles that do not meet the inclusion criteria were excluded. A total of 198 works were found in full; of these, 3 articles were duplicated in the search strategies, thus totaling 8 selected. Results and Discussion: It was found that it is necessary to analyze the morphophysiological changes that can be found with the use of mini-implants. It was also observed that the size of mini-implants influences the increase in mechanical anchorage, as well as the incidence of the paranasal sinuses, concluding that the main determining factors in bone anchorage are bone density and placement depth. The use of miniimplants in the palate does not interfere with tooth movement, in addition to having enough bone, enabling the insertion of the mini-implant. The authors also report that there are multiple reasons for a mini-implant failure or complete loss, such as periodontitis and peri-implantitis, causing soft and hard tissue infection, especially when inadequate oral hygiene or poor post-surgical control persists. Final considerations: The knowledge and mastery of the anatomical structures of the head and neck, as well as the morphophysiological changes can lead to the implantation of orthodontic miniimplants, contributes to the achievement of clinical success, reducing side effects and the treatment time is reduced.
\end{abstract}

Keywords: Anatomy; Mini-implants; Orthodontics.

\title{
Resumen
}

Introducción: Los miniimplantes representan un gran avance en Odontología para ayudar en el tratamiento de ortodoncia, también llamados microimplantes y los micro tornillos se conocen como dispositivos de anclaje temporal. Objetivos: Realizar una revisión integradora de la literatura, basada en el análisis de hitos anatómicos, enfatizando la importancia del estudio de la anatomía a la hora de colocar miniimplantes. Metodología: Se trata de una revisión integradora de la literatura de carácter cualitativo basada en la pregunta orientadora: ¿Qué cambios morfofisiológicos interfieren con el éxito de los miniimplantes en los tratamientos de ortodoncia? La base de datos fue: Biblioteca Nacional de Medicina de EE. UU. (NLM - PubMed), utilizando los siguientes descriptores (MeSHterms): Anatomía; Miniimplantes. Los criterios de inclusión se publicaron en inglés, portugués y español; publicaciones entre enero de 2000 y enero de 2021 y que abordó el tema de manera satisfactoria. Se excluyeron los artículos que no cumplen con los criterios de inclusión. Se encontraron un total de 198 obras completas; de estos, 3 artículos se duplicaron en las estrategias de búsqueda, totalizando 8 seleccionados. Resultados y Discusión: Se encontró que es necesario analizar los cambios morfofisiológicos que se pueden encontrar con el uso de miniimplantes. También se observó que el tamaño de los miniimplantes influye en el aumento del anclaje mecánico, así como en la incidencia de los senos paranasales, concluyendo que los principales determinantes en el anclaje óseo son la densidad ósea y la profundidad de colocación. El uso de miniimplantes en el paladar no interfiere con el movimiento dentario, además de tener suficiente hueso, permitiendo la inserción del miniimplante. Los autores también informan que existen múltiples razones para la falla o pérdida completa de un miniimplante, como periodontitis y periimplantitis, que causan infección de tejidos duros y blandos, especialmente cuando persiste una higiene bucal inadecuada o un control posquirúrgico deficiente. Consideraciones finales: El conocimiento y dominio de las estructuras anatómicas de la cabeza y el cuello, así como los cambios morfofisiológicos pueden conducir a la implantación de mini-implantes de ortodoncia, contribuye al logro del éxito clínico, reduciendo los efectos secundarios y el tiempo de tratamiento es reducido.

Palabras clave: Anatomía; Mini-implantes; Ortodoncia.

\section{Introdução}

A utilização de mini-implantes para auxiliar no tratamento ortodôntico representa um avanço nessa área da odontologia.

Anteriormente, o processo de ancoragem era realizado com os próprios dentes do paciente, resultando em diversos efeitos colaterais (Lima et al., 2010; Marcello-Machado et al., 2018). Atualmente, o uso de mini-implantes para ancoragem em 
ortodontia implica na diminuição de tais efeitos. Esse fato é explicado pela existência de reduzidos e variados tamanhos dos mini-implantes, que possibilitam sua instalação em regiões estratégicas, a realização de movimentos complexos, como a intrusão, além de uma maior previsibilidade da movimentação e redução do tempo de tratamento (Reyenders \& Cacciatore, 2016; Gurdán \& Szalma, 2018; Enkling et al., 2019).

Também denominados de micro-implantes, microparafusos ou parafusos de ancoragem, os mini-implantes são conhecidos como dispositivos de ancoragem temporária (DAT) (Garg \& Gupta, 2015; Leo et al., 2016). Sendo assim, para que haja ancoragem absoluta é necessária sua adaptação mecânica à estrutura óssea previamente mineralizada, mecanismo chamado de intertravamento que, nesses casos, ocorre em região de maxilas e mandíbula (Consolaro \& Romano, 2014; Di Leonardo et al., 2018).

As maxilas e mandíbula são ossos importantes para fixação e suporte de músculos, tendões, dentes e tecidos moles, que estão associados às funções da cabeça e do pescoço (Lee, Moon \& Hong, 2017). Nesse sentido, a anatomia desses ossos apresenta distintas espessuras, densidade, volume e organização das estruturas ósseas, que variam de acordo com a adaptação às condições individuais de cada pessoa (Consolaro \& Romano, 2014). Dessa forma, para que haja uma instalação de mini-implantes bem sucedida, é importante que o cirurgião dentista tenha domínio acerca das características anatômicas envolvidas.

Portanto, este artigo científico teve como objetivo realizar uma revisão integrativa da literatura, com ênfase na análise dos marcos anatômicos e enfatizar a importância do estudo da anatomia de cabeça e pescoço, precisamente dos ossos Maxilares e Mandíbula, na colocação de mini-implantes ortodônticos.

\section{Metodologia}

Esta revisão integrativa da literatura possui uma metodologia qualitativa, sendo baseada em Rother (2007) e Pereira et al. (2018), e no desenvolvimento da seguinte pergunta de pesquisa: Quais alterações morfofisiológicas interferem no sucesso de mini-implantes em tratamentos ortodônticos?

Para isto, foi utilizada a base de dados eletrônica: U. S. National Library of Medicine (PubMed) para pesquisar e identificar estudos que respondessem à pergunta norteadora desta revisão integrativa da literatura. A base de dados foi pesquisada para estudos realizados entre janeiro de 2000 a maio de 2021.

Esta revisão integrativa baseou-se em cinco etapas: Na primeira etapa foi o estabelecimento dos descritores para ambas as bases de dados, sendo uma com a utilização de MeSHterms (PubMed). Em seguida, segunda etapa, fora feito a busca avançada nas bases e análise do quantitativo dos artigos científicos presentes na íntegra. Logo em seguida, na terceira etapa, foram selecionados os artigos que se adequaram aos critérios de elegibilidade estabelecidos pelos pesquisadores. Na quarta e quinta etapa, os pesquisadores formularam uma tabela descritiva sobre os autores, objetivo da pesquisa, protocolo, resultados e conclusão e em seguida, desenvolvimento da discussão dos artigos científicos para uma análise do melhor manejo do clareamento dental, a fim de se chegar ao sucesso clínico e responder à pergunta norteadora estabelecida no início desta metodologia.

Foram utilizados dois descritores para a composição da chave de pesquisa, sendo os seguintes (MeSH): Anatomy; Miniimplants. Em seguida, os pesquisadores selecionaram os trabalhos com análise no título e resumo, com base nos critérios de elegibilidade. Os critérios de elegibilidade foram os seguintes: artigos publicados em inglês, português e espanhol; publicações entre janeiro de 2000 e maio de 2021; artigos que se adequem à temática.

Também foi utilizado o sistema de formulário avançado para busca e seleção dos artigos utilizando conector booleano “AND”. Em seguida, artigos que preencheram os critérios de elegibilidade foram identificados e incluídos na revisão. 


\section{Resultados e Discussão}

Os trabalhos que preencheram todos os critérios de seleção foram incluídos no estudo, os que não preencheram os critérios e/ou não se mostraram relevantes foram excluídos. Os resultados por análise foram representados na Tabela 1:

Tabela 1 - Seleção dos artigos por análise empregada e estabelecimento dos critérios de inclusão.

\begin{tabular}{cccc}
\hline & Íntegra & Duplicados & Artigos selecionados \\
\hline PubMed & 198 & 3 & 8
\end{tabular}

Fonte: Autores (2021).

Portanto, a partir dessa estratégia de busca, foram encontrados ao todo 198 trabalhos na íntegra; destes, 3 artigos encontravam-se duplicados nas estratégias de busca, totalizando, assim, 8 selecionados.

Atualmente, há questionamentos sobre quais alterações morfofisiológicas podem influenciar para o sucesso dos miniimplantes ortodônticos, sejam elas extrínsecas ou intrínsecas ao indivíduo (Gurdán \& Szalma, 2018). Dessa forma, é necessária a análise das alterações morfofisiológicas que podem ser encontradas com a utilização de mini-implantes. Para isso, estabeleceuse a construção da Tabela 2 aos estudos clínicos selecionados, com formulação das colunas (Autor/Ano; Objetivo do estudo; Protocolo; Resultados; Conclusão).

Tabela 2 - Relatos de casos detalhados em tabela de resultados.

\begin{tabular}{|c|c|c|c|}
\hline Autor/Ano & Objetivo do estudo & Alterações morfofisiológicas encontradas & Conclusão \\
\hline $\begin{array}{l}\text { Arcuri et al., } \\
2007 .\end{array}$ & $\begin{array}{l}\text { Descrever a experiência na } \\
\text { utilização de mini-implantes } \\
\text { para ancoragem ortodôntica } \\
\text { durante cinco anos e fazer } \\
\text { uma avaliação crítica acerca } \\
\text { dos resultados obtidos com } \\
\text { esse método. }\end{array}$ & $\begin{array}{l}\text { Todos os mini-implantes foram osteointegrados com } \\
\text { sucesso, exceto um que foi perdido por condições } \\
\text { críticas de higiene. Dois implantes foram substituídos } \\
\text { por causa das forças da língua, que interferiu na } \\
\text { osteointegração imediatamente após a cirurgia. }\end{array}$ & $\begin{array}{l}\text { Os mini-implantes } \\
\text { demonstraram ser de fácil } \\
\text { utilização de forma auxiliar e } \\
\text { eficiente em ortodontia quando } \\
\text { há necessidade de ancoragem } \\
\text { absoluta. }\end{array}$ \\
\hline $\begin{array}{l}\text { Apel et al., } \\
2009\end{array}$ & $\begin{array}{l}\text { Investigar a microflora } \\
\text { associada a mini-implantes } \\
\text { com falha vs. sucesso. }\end{array}$ & $\begin{array}{l}\text { Dos } 25 \text { pacientes, cinco apresentaram falha. Do total de } \\
76 \text { mini-implantes, oito foram retirados por motivo de } \\
\text { mobilidade. A taxa de falha foi, portanto, de } 10,5 \% \text {. A } \\
\text { análise bacteriana quantitativa de mini-implantes com } \\
\text { falha }(\mathrm{n}=8) \text { mostrou um número médio de células de }\end{array}$ & $\begin{array}{l}\text { O sulco peri-implantar ao redor } \\
\text { dos mini-implantes ortodônticos } \\
\text { fracas-sados não apresentou } \\
\text { uma flora bacteriana agressiva } \\
\text { específica. }\end{array}$ \\
\hline
\end{tabular}
$1,6 \times 10^{7}$. Claramente, as espécies clássicas de origem oral periodontopatogênica ou peri-implantite, como A. actinomycetemcomitans e P. gingivalis, estavam ausentes ou raramente encontradas ( $\mathrm{T}$. forsythensis) nos casos e, como esperado, estavam ausentes nos controles. No entanto, o designado 'patógeno' $\mathrm{T}$. denticola estava frequentemente presente em ambos os casos (50\%) e controles $(75 \%)$, demonstrando quão fraco pode ser o valor preditivo positivo desta espécie para doenças gengivais. Além disso, espécies com reconhecido papel principalmente fisiológico na cavidade oral, como $A$. odontolyticus, S. gordonii, S. mitis e V. parvula, estiveram presentes em todas as amostras analisadas, independentemente de serem casos ou controles. Com as 10 espécies restantes, os resultados foram mais heterogêneos, mas $C$. gingivalis, E. corrodens e $F$. nucleatum foram frequentemente ou sempre encontrados em ambos os grupos.

Lemieux et O objetivo deste trabalho foi al., 2011. caracterizar o padrão de colocação e os fatores que influenciam a estabilidade
A profundidade de colocação foi fortemente dependente do comprimento do mini-implante: $15 \%$ dos implantes de $6 \mathrm{~mm}$ não conseguiram ancorar suas seções paralelas no osso cortical, mas $95 \%$ das seções paralelas do mini-
Durante a seleção do comprimento do mini-implante, o clínico deve considerar o importante equilíbrio entre a 

em cadáveres humanos. Os fatores estudados foram o comprimento do miniimplante, profundidade de colocação, densidade óssea e tipo de osso.

$\begin{array}{ll}\text { Omasa } \text { et } & \text { O objetivo deste estudo foi } \\ \text { al., 2012. } & \text { investigar os efeitos } \\ & \text { estimuladores da terapia a } \\ & \text { laser de baixa potência } \\ & \text { (LLLT) sobre a estabilidade } \\ & \text { de mini-implantes em tíbias } \\ & \text { de ratos. }\end{array}$
de ratos. primária de mini-implantes
Avaliar a relação entre a mobilidade do miniimplante durante a fase de cicatrização e o prognóstico para a estabilidade do implante. corticais placas, atingindo o osso cortical lingual. Mini-

Os valores de Periotest (PTVs) foram significativamente

$\begin{array}{ll}\text { al., 2012. } & \text { mobilidade do mini- } \\ & \text { implante durante a fase de } \\ & \text { cicatrização e o prognóstico } \\ & \text { para a estabilidade do } \\ & \text { implante. }\end{array}$

Agrawal et Relatar a formação de al. (2013) exostoses do osso alveolar durante uma atividade ortodôntica tratamento com mini implantes, discutindo algumas características desta condição clínica, juntamente com a gerência. implante de $10 \mathrm{~mm}$ penetraram além da cortical vestibular; todas as 20 pontas de $6 \mathrm{~mm}$, os miniimplantes (100\%) atingiram o osso esponjoso, enquanto $75 \%$ dos implantes de $10 \mathrm{~mm}$ penetraram ambas as implantes mais longos foram associados a maiores incidências de perfurações sinusais e bicorticais. Os coeficientes de correlação entre o máximo inicial mecânico força de ancoragem e os fatores estudados foram os seguintes: densidade óssea e profundidade de colocação combinados $(\mathrm{r}=0,65, \mathrm{P}<0.001)$, comprimento do mini-implante $(\mathrm{r}=0,45, \mathrm{P}=0,004)$, densidade óssea $(\mathrm{r}=0,42, \mathrm{P}=0,007)$ e profundidade de colocação $(r=0,29, P=0,06)$. menores no grupo LLLT do que no grupo de controle nos dias 7 ( 0,79 vezes) e 35 ( 0,67 vezes; <p 0,05). PTVs foram significativamente menores no dia 35 do que no dia 7 em ambos os controles $(0,55$ vezes) e LLLT $(0,47$ vezes) <grupos (p 0,05). As imagens R_mCT revelaram ossificação peri-implantar ao longo do tempo. Tecido ósseo recém-formado foi observado ao redor dos miniimplantes em ambos os grupos logo 5 dias após a cirurgia, e a formação óssea pareceu ser mais forte no grupo LLLT. Imagens mostraram resultados semelhantes aos de imagens R_mCT. Nenhuma diferença significativa no volume ósseo foi observada entre os grupos controle e LLLT nos dias 0,3 ou 5. No entanto, o volume ósseo foi 1,53 vezes maior no grupo LLLT do que no grupo controle no dia 7. A expressão do gene BMP-2 foi 1,92 vezes maior no grupo LLLT $<$ do que no grupo controle (p 0,05) logo 1 dia após LLLT. Nenhuma diferença significativa na expressão de BMP-2 foi observada entre os grupos nos dias 3, 5 e 7. Com os orifícios de $1,1 \mathrm{~mm}$ de diâmetro, foi observado menos suporte ósseo ao redor do mini-implante e muitas lacunas estavam presentes entre o osso e o miniimplante, resultando em ancoragem insuficiente. O Valor de Periotest no grupo de orifícios de $0,8 \mathrm{~mm}$ foi menor do que para os grupos de 0,9 e $1,0 \mathrm{~mm}$, refletindo o índice de contato osso-implante reduzido. Isso pode estar relacionado à cicatrização retardada causada por estresse excessivo ou pressão no osso pelos tecidos circundantes durante a inserção, quando o orifício é muito pequeno em comparação com o diâmetro do implante. A relação implante / diâmetro do orifício no grupo de $1,1 \mathrm{~mm}$ foi de 84,61 por cento quando o diâmetro de $1,3 \mathrm{~mm}$ foi assumido a meio caminho entre o tamanho máximo e mínimo do mini-implante, o que pode resultar em formação óssea insuficiente e um miniimplante instável. Portanto, a melhor relação diâmetro / mini-implante deve estar entre 69 e 77 por cento, os valores mais baixos foram 62 e 84 por cento para os mini-implantes com um diâmetro de $1,3 \mathrm{~mm}$.

ancoragem e o risco de complicações ou danos na colocação para os tecidos. Miniimplantes mais longos permitem mais ancoragem, no entanto, eles estão associados a um maior risco de danos às estruturas vizinhas. Profundidade $\mathrm{de}$ colocação e densidade óssea no local de colocação do miniimplante são os melhores preditores de estabilidade primária.

LLLT aumentou a estabilidade dos mini-implantes colocados na tíbia de ratos e acelerou a formação óssea peri-implantar através do aumento da expressão gênica de BMP-2 nas células vizinhas. Portanto, é mais provável que quando os miniimplantes são colocados no osso alveolar de pacientes adolescentes, o LLLT ao redor dos mini-implantes aumenta sua estabilidade.

Para obter estabilidade do miniimplante, o diâmetro do orifício deve estar entre 69 e 77 por cento do diâmetro do miniimplante para mini-implante de $1,3 \mathrm{~mm}$ de diâmetro. Uma diminuição significativa na mobilidade do mini-implante após 3 semanas, testado com o periotest, implica um bom prognóstico para estabilidade subsequente.

$\mathrm{O}$ tratamento ortodôntico formulado incluiu as extrações de todos os primeiros pré-molares para retrair os dentes anteriores superiores e inferiores e para intrometer o segmento anterior maxilar usando mini implantes ortodônticos para reduzir a gengiva sorriso devido ao excesso maxilar. Após alinhamento, um mini implante ortodôntico $(1,2 \mathrm{~mm}$ de diâmetro e $8 \mathrm{~mm}$ de comprimento) foi colocado entre as raízes do maxilar incisivos centrais e laterais de ambos os lados. Força intrusiva foi aplicada ao segmento anterior, anexando uma mola espiral fechada Niti (força média) do mini implante ao arco rígido de $0,01900 \times 0,02500$ SS. Após cerca de 4 meses de intrusão, a abertura da mordida foi
A cirurgia óssea de ressecção foi planejada para o paciente. Após 7 dias, o paciente foi avaliado quanto ao desconforto pósoperatório e a cura, que transcorreu sem intercorrências. Instruções de higiene oral foram reforçados e 1 mês de terapia periodontal de suporte foi agendado. Após 1 mês, a terapia ortodôntica ativa foi continuada 


$\begin{array}{ll}\text { Garg \& } & \text { Avaliar a mobilidade de } \\ \text { Gupta, } & \text { mini-implantes ortodônticos } \\ \text { 2015. } & \text { sob carga ortodôntica por } \\ & \text { meio de tomografia } \\ & \text { computadorizada }\end{array}$

alcançada e houve redução na quantidade de gengiva visível no sorriso. No entanto, intraoral exame revelou a presença de inchaços ósseos duros e indolores na superfície vestibular da gengiva inserida na parte superior dentes anteriores. Mini-implantes foram removidos e o paciente foi encaminhado ao periodontista para avaliação e manejo do quadro. Depois de avaliar o paciente (caso história, exame clínico e radiológico) foi feito o diagnóstico provisório de exostose do osso alveolar.

: Em média, os miniparafusos foram extrudados e inclinados para frente significativamente, de $1 \mathrm{~mm}$ na cabeça do parafuso no plano axial (Grupo III) e 0,728 mm no plano coronal (Grupo IV). A cauda dos miniimplantes apresentou inclinação média de $0,567 \mathrm{~mm}$ no plano axial (Grupo I) e $0,486 \mathrm{~mm}$ no plano paraxial (Grupo V). A menor mobilidade média foi demonstrada pelo corpo do parafuso de $0,349 \mathrm{~mm}$ no plano axial (Grupo II). Clinicamente, nenhuma mobilidade significativa foi observada.

e nenhuma recidiva foi observada depois disso.

Os miniparafusos são uma ancoragem estável para a movimentação dentária ortodôntica, mas não permanecem absolutamente estacionários como um implante endósseo durante a carga ortodôntica, embora os miniparafusos possam se mover de acordo com o local de colocação, carga ortodôntica e inflamação do tecido periimplantar.

Gurdán \& O objetivo deste trabalho foi Existem vários problemas críticos que devem ser Szalma, calcular as taxas de sucesso 2018. e complicações dos miniimplantes ortodônticos. considerados para prevenir complicações nos miniimplantes, um desses problemas é o local de inserção. Durante a pesquisa, o carregamento do parafuso foi realizado imediatamente após as inserções, mantendo as forças de tensão abaixo de $150 \mathrm{~g}$. Assim, após a inserção do mini implante, foram registradas infecções de tecidos moles e ossos, mobilidade do implante e perda do parafuso, fratura do implante e lesão do dente vizinho.
A taxa geral de sucesso dos mini-implantes foi considerada aceitável neste estudo, no entanto, a mobilidade do parafuso na prega vestibular apresentou uma alta incidência, sugerindo a consideração completa da carga imediata pelos mini-implantes bucais.

Fonte: Autores (2021).

Arcuri et al (2007) avaliaram a utilização de mini-implantes palatino para ancoragem ortodôntica durante cinco anos, analisando os resultados obtidos. Na cavidade oral o palato é uma área que não apresenta interferência nos movimentos dentários, além de haver osso suficiente, possibilitando a inserção do mini-implante. Assim, os implantes foram osteointegrados com sucesso, apresentando resultados satisfatórios, no entanto, o período de osteointegração dos mini-implantes pode aumentar o tempo de tratamento ortodôntico. Dessa forma, a utilização de mini-implantes palatino demonstra-se como um método eficiente que permite estabilidade primária e facilita a ortodontia quando a ancoragem absoluta é necessária, sendo de fácil utilização. Logo, após a osteointegração completa, inicia-se a fase ortodôntica e após a finalização do tratamento o implante palatino é removido.

No que concerne à pesquisa de Apel et al. (2009), os autores relataram que existem múltiplas razões para a falha ou a perda completa de um mini implante. No entanto, mesmo colocados transgengivalmente estão em contato direto com todos os tipos de bactérias orais que implicam na periodontite e peri-implantite, com capacidade de penetrar ao lado do mini-implante, causando infecção de tecidos moles e duros, especialmente quando a higiene oral inadequada ou controle pós-cirúrgico deficiente persiste. Em relação a aspectos anatômicos, uma das principais causas de falha se encontra na proximidade da raiz, além disso, a densidade óssea também foi apontada como um indicador com risco para falha. Em casos clínicos malsucedidos, a razão para a perda do implante permanece obscura, mas os autores descrevem no seu estudo que os mini-implantes falhados não mostram uma flora bacteriana agressiva especifica na região de sulco peri-implantar.

Em um estudo realizado por Lemieux et al (2010), foi analisado padrões relacionados a colocação de mini-implantes a partir de tomografias computadorizadas. Nesse estudo, concluiu-se que, o tamanho dos mini-implantes influencia no aumento 
da ancoragem mecânica, assim como na incidência dos seios paranasais e perfuração bicortical. Portanto, os principais fatores determinantes na ancoragem óssea foram, em ordem crescente: densidade óssea e profundidade de colocação, respectivamente.

O estudo realizado por Omasa et al., (2012) buscou avaliar os efeitos que os estimuladores da terapia a laser de baixa potência têm sobre a estabilidade de mini-implantes em tíbias de ratos. Observou-se, a partir da avaliação dos resultados do teste de estabilidade e R_mCT, que houve aumento da estabilidade dos mini-implantes, ocasionado pelo aumento da formação óssea peri-implantar. Também se observou que o volume ósseo no grupo LLLT foi 1,53 vezes maior do que no grupo controle no dia 7. Portanto, quando os mini-implantes são colocados no osso alveolar de pacientes adolescentes, é mais provável que o LLLT ao redor dos mini-implantes aumente sua estabilidade.

Um estudo realizado por Uemura et al. (2011) avaliou a relação entre a mobilidade do mini-implante durante a fase de cicatrização e o prognóstico para a estabilidade do implante. Para isto, investigou os diâmetros de orifícios e de mini-implantes, estimando o sucesso do procedimento a partir da análise da mudança na mobilidade ao longo do tempo. Esses valores eram obtidos por meio de periotests medidos imediatamente após a inserção do mini-implante, observando-se que, no prazo de 3 semanas, esses valores foram reduzidos em orifícios que possuíam o tamanho correto, conferindo maior estabilidade ao miniimplante, todavia, aumentaram em orifícios incorretos. Partindo disso, o estudo ressaltou que um período suficiente de cicatrização contribui para o sucesso da estabilidade do mini-implante e que orifícios com diâmetros muito pequenos em relação ao implante estão sujeitos a fatores como estresse ou pressão exercida sobre os tecidos circundantes na inserção do mini-implante, que incidem no retardamento da cicatrização.

Agrawal et al. (2013) relataram a formação de exostoses no osso alveolar durante tratamento ortodôntico com a utilização de mini-implantes, em que, após 4 meses do início do tratamento, foram encontrados inchaços ósseos duros e indolores na superfície vestibular da gengiva inserida na parte superior dos dentes anteriores. Sendo assim, com o diagnóstico provisório de exostose do osso alveolar, foi estabelecida uma cirurgia óssea de ressecção como tratamento para tais alterações, em que o trans e o pós-operatório transcorreu sem intercorrências, sem haver recidivas da exostose.

Na pesquisa de Garg \& Gupta (2015), os autores avaliaram a mobilidade de mini-implantes ortodônticos sob carga ortodôntica por meio de tomografia computadorizada. Os mini-parafusos foram utilizados como acessórios temporários para movimentação dentária ortodôntica e removidos no final do tratamento, com esse estudo observou-se que em média, os miniparafusos foram extrudados e inclinados para frente significativamente, $1 \mathrm{~mm}$ na cabeça do parafuso no plano axial, 0,728mm no plano coronal, enquanto na cauda dos mini-implantes teve uma inclinação média de $0,567 \mathrm{~mm}$ no plano axial e $0,486 \mathrm{~mm}$ no plano paraxial. A menor média de mobilidade foi demonstrada pelo corpo do parafuso de $0,349 \mathrm{~mm}$ no plano axial e clinicamente nenhuma mobilidade significativa foi observada. Portanto, os miniparafusos são uma ancoragem estável para a movimentação dentária ortodôntica, mas não permanecem absolutamente estacionários, isso não é um problema se os efeitos do tratamento forem alcançados. Porém, o deslocamento dos mini-implantes seria um problema sério quando o descolamento prejudica órgãos vitais adjacentes, como as raízes dos dentes, os nervos e os vasos sanguíneos, logo, eles não devem ser colocados em um local adjacente a órgãos vitais.

Gúrdan e Szalma (2018) afirmam que vários problemas críticos devem ser considerados para prevenir complicações dos mini-implantes. Um desses problemas é o local de inserção, principalmente os que não são inseridos na mucosa queratinizada se soltam numa proporção maior. Foi observado também que pontos de inflamação foi mais frequentemente detectado em mini parafusos inseridos na face vestibular, devido ao impacto das forças musculares e à proporção variável de gengiva inserida. Além disso, a estabilidade do mini parafuso é influenciada pela qualidade e quantidade do osso cortical, quanto mais fino for o osso, a ancoragem esquelética não será garantida. Dessa forma, os resultados do estudo indicaram que a perda do mini implante é mais frequente na área vestibular devido à espessura cortical do que a área do palato. 


\section{Considerações Finais}

O uso de mini-implantes no tratamento ortodôntico pode contribuir para o alcance do sucesso clínico, visto que o seu uso implica na diminuição de efeitos colaterais, bem como o tempo de tratamento é reduzido. Tendo em vista os estudos incluídos nessa revisão integrativa da literatura, notou-se a importância do conhecimento anatômico e de alterações morfológicas relacionados ao sucesso ou não no uso de mini-implantes no tratamento ortodôntico.

Assim, para o tratamento ser eficaz, é fundamental que o profissional tenha domínio das estruturas anatômicas de cabeça e pescoço, além de saber quais alterações morfofisiológicas podem influenciar na implantação dos mini-implantes ortodônticos, para assim, proporcionar ao paciente um bom resultado clínico. Novas pesquisas são necessárias para a determinação dos pontos focais de colocação dos mini-implantes. Os resultados dessa revisão responderam à pergunta norteadora estabelecida na metodologia.

\section{Referências}

Agrawal, N. et al. (2013). Alveolar bone exostoses subsequent to orthodontic implant placement. BMJ Case Reports, DOI: 10.1136/ bcr-2012-007951.

Apel, S. et al. (2009). Microflora associated with successful and failed orthodontic mini-implants. Clinical Oral Implants Research, 20(11): 1186-1190.

Arcuri, C. et al. (2007). Five years of experience using palatal mini-implants for orthodontic anchorage. Journal Oral Maxillofacial Surgery, 65 (12): $2492-2497$.

Consolaro, A. \& Romano, F.L. (2014). Reasons for mini-implants failure: choosing installation site should be valued! Dental Press Journal Orthodontics, Maringá, 19(2): 18-24.

Di Leonardo, B. et al. (2018). Insertion torque values and success rates for paramedian insertion of orthodontic mini-implants: A retrospective study. Insertionsdrehmoment und Erfolgsrate paramedian inserierter kieferorthopädischer Mini-Implantate : Eine retrospektive Studie. Journal of orofacial orthopedics = Fortschritte der Kieferorthopadie: Organ/official journal Deutsche Gesellschaft fur Kieferorthopadie, 79(2), 109-115.

Enkling, N. et al. (2019). A prospective cohort study on survival and success of one-piece mini-implants with associated changes in oral function: Five-year outcomes. Clinical oral implants research, 30(6), 570-577.

Garg, K.K. \& Gupta, M. et al. (2015). Avaliação da estabilidade de mini-implantes ortodônticos sob carga ortodôntica: um estudo de tomografia computadorizada. Indian Journal of Dental Research, 26(3): 237-43.

Gurdán, Z. \& Szalma, J. (2018). Evaluation of the success and complication rates of self-drilling orthodontic mini-implants. Nigerian Journal of Clinical Practice, 21(5):546-52.

Lee, R. J., Moon, W., \& Hong, C. (2017). Effects of monocortical and bicortical mini-implant anchorage on bone-borne palatal expansion using finite element analysis. American journal of orthodontics and dentofacial orthopedics: official publication of the American Association of Orthodontists, its constituent societies, and the American Board of Orthodontics, 151(5), 887-897.

Lemieux, G. et al. (2010). Computed tomographic characterization of mini-implant placement pattern and maximum anchorage force in human cadavers. American Association of Orthodontists, 140 (3): 356-65.

Leo, M. et al. (2016). Temporary anchorage devices (TADs) in orthodontics: review of the factors that influence the clinical success rate of the mini-implants. La Clinica terapeutica, 167(3), e70-e77.

Lima, L.A.C. et al. (2010). Mini-implante como ancoragem absoluta: ampliando os conceitos de mecânica ortodôntica. Innovations Implant Journal: Biomaterials and Esthetics (Online), 5(1): 85-91.

Marcello-Machado, R. M. et al. (2018). Mini-implants and narrow diameter implants as mandibular overdenture retainers: A systematic review and meta-analysis of clinical and radiographic outcomes. Journal of oral rehabilitation, 45(2), 161-183.

Omasa, S. et al. (2012). Low-Level Laser Therapy Enhances the Stability of Orthodontic Mini-Implants via Bone Formation Related to BMP-2 Expression in a Rat Model. Photomedicine and Laser Surgery, 30(5): 255-261.

Pereira, A.S. et al. (2018). Metodologia da pesquisa científica. [e-book]. Santa Maria. Ed. UAB/NTE/UFSM.

Reynders, R. M., \& Cacciatore, G. (2016). No confidence that success rates of self-drilling and self-tapping insertion techniques of orthodontic mini-implants are similar. Evidence-based dentistry, 17(4), 111-113.

Rother, E.T. (2007). Revisão sistemática X revisão narrativa. Acta paulista de Enfermagem; 20(2):v.

Souza, M.C.; Silva, M.D. \& Carvalho, R. (2010). Revisão integrativa: O que é e como fazer? Einstein, 8(1): 102-6.

Uemura, M. et al. (2012). Orthodontic mini-implant stability and the ratio of pilot hole implant diameter. European Journal of Orthodontics, $34(1)$ : 52-56. 